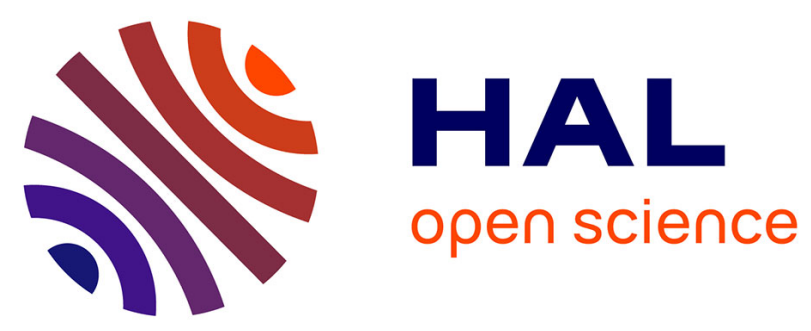

\title{
Dissolution mechanisms of wood cellulose fibres in $\mathrm{NaOH}$-water
}

\author{
Nicolas Le Moigne, Patrick Navard
}

\section{To cite this version:}

Nicolas Le Moigne, Patrick Navard. Dissolution mechanisms of wood cellulose fibres in NaOH-water.

Cellulose, 2010, 17 (1), pp.Pages 31-45. 10.1007/s10570-009-9370-5 . hal-00509589

\section{HAL Id: hal-00509589}

https://hal-mines-paristech.archives-ouvertes.fr/hal-00509589

Submitted on 29 Apr 2011

HAL is a multi-disciplinary open access archive for the deposit and dissemination of scientific research documents, whether they are published or not. The documents may come from teaching and research institutions in France or abroad, or from public or private research centers.
L'archive ouverte pluridisciplinaire HAL, est destinée au dépôt et à la diffusion de documents scientifiques de niveau recherche, publiés ou non, émanant des établissements d'enseignement et de recherche français ou étrangers, des laboratoires publics ou privés. 


\title{
Dissolution mechanisms of wood cellulose fibres in $\mathrm{NaOH}$-water
}

\author{
Nicolas Le Moigne and Patrick Navard * \\ Mines ParisTech, CEMEF - Centre de Mise en Forme des Matériaux, CNRS UMR \\ 7635, BP 207, 1 Rue Claude Daunesse, 06904 Sophia Antipolis Cedex, France \\ Member of the European Polysaccharide Network of Excellence (EPNOE), \\ www.epnoe.eu
}

(*) Corresponding author. Telephone: 33(0)493957466. Fax: 33(0)492389752. Email: patrick.navard@mines-paristech.fr

\begin{abstract}
Four wood pulps and a microcrystalline cellulose were dissolved in a $\mathrm{NaOH} 8 \%$ - water solution. Insoluble fractions and clear solution fractions were isolated by centrifugation and were observed by optical microscopy and transmission electron microscopy. Molecular weight distribution, carbohydrate composition and cellulose II content were measured. The dissolution of wood cellulose fibres in $\mathrm{NaOH} 8 \%$ - water solutions occurs by successive dismantlement and fragmentation steps governed by the swelling and the shearing of the original structure. The cellulose from insoluble and clear solution fractions is in both case converted in cellulose II and the insoluble fractions contain embedded mannans. Besides, the molecular weight distributions of cellulose from insoluble and clear solution fractions reveal the existence of heterogeneities in dissolution capacity of the cellulose chains, independent to the degree of polymerization, which are related to the chemical environment of the chains in the fibre structure.
\end{abstract}

Keywords. Cellulose, wood, fibre, solution, dissolution, $\mathrm{NaOH}$, structure

\section{Introduction}

The dissolution of cellulose is of great interest and it has been studied in numerous solvents for over a century. However, some of the mechanisms involved in the dissolution at the different structural levels of cellulose fibre are still not well understood and controlled. First evidences of the complex swelling and dissolution of cellulose fibres such as the ballooning phenomenon were observed and discussed long ago (Nägeli 1864; Pennetier 1883) and were followed by several studies over the last century (e.g.: Flemming and Thaysen 1919; Hock 1950; Hock 1954; Rollins and Tripp 1954; Tripp and Rollins 1952). More recent studies (Chanzy et al. 1983; Cuissinat and Navard 2006a) have shown that the dissolution mechanisms of cellulose fibres are strongly dependent on the solvent quality. The last authors identified four main dissolution modes for 
wood and cotton fibres as a function of the quality of the solvent by using $\mathrm{N}$ methylmorpholine $N$-oxide (NMMO) with various amounts of water. The same mechanisms were also observed when using solvents as $\mathrm{NaOH}$-water with or without additives (Cuissinat and Navard 2006b), ionic liquids (Cuissinat et al. 2008a) and other chemicals (Cuissinat 2006) for a wide range of plant fibres (Cuissinat and Navard 2008) and some cellulose derivatives that were prepared without dissolution (Cuissinat et al. 2008b). From all these studies, it was shown that the key parameter in the dissolution mechanism is the morphology of the fibre: as long as the original wall structure of the native fibre is preserved, the dissolution mechanisms are mostly similar for wood, cotton, other plant fibres and some cellulose derivatives. In a recent paper (Le Moigne et al. 2008), we demonstrated the existence of a centripetal radial gradient in the dissolution capacity within the fibre. The older was the cellulose deposition during the biosynthesis, the more difficult it is to dissolve it. These results can be related to age-dependent structural re-organisations in the cell wall layers and / or to the presence of non-cellulosic networks in the outside walls. All these features originate from the succession of polymers and products depositions and their deformation during the extension and thickening of the plant cell.

Dissolution or chemical treatments of cotton fibres and wood pulps are not easy. As far as dissolution is concerned, a lot of reports suggest that the classical thermodynamic parameters, i.e. concentration and molecular weights, are not of primary importance (they are of course playing their usual thermodynamic role). Crystallinity does not seem also to play a major role for solubility. At least at rather low molar mass and in situations where the structure is not hampering dissolution, crystalline and amorphous parts are dissolving in about the same way. As was suggesting by Isogai and Atalla (1998), the origin of the difficulty of dissolving cellulose fibres should be searched in an unknown long range ordering or interaction that must be disrupted before being able to fully dissolve high molar mass pulp. This conclusion came after remarking that pulps that were not dissolving in a bad solvent ( $\mathrm{NaOH}$-water) were soluble after full dissolution in another solvent and regeneration. This assumption of a long range organisation to be removed to facilitate dissolution is in agreement with recent works where it was showed that placing a cotton fibre or a regenerated fibre under tension is preventing full dissolution while the solvent was able to fully access the cellulose 
chains under tension, thus eliminating the classical "accessibility of the chain" reason for the lack of reactivity (Le Moigne 2008). The hypothesis brought by the authors is that, to be dissolved, chains must perform conformational movements that are not allowed if the whole polymer chains is blocked into a long range fixed H-bond network.

Activation is the usual term for describing treatments that increase cellulose reactivity. Aside obvious physical changes that are indeed helping reactivity, some activation treatments may include the destruction of these long range interactions that must be removed to have reactive cellulose fibres. In a comprehensive study, Yamashiki et al. (1990a, b, c) demonstrated the possibility to improve the alkaline solubility of wood pulps by applying a steam explosion treatment. They found a strong correlation between the alkaline solubility and the degree of breakdown of the intramolecular hydrogen bonds attached to the $\mathrm{C} 3$ and the C6 carbon position of the anhydroglucose units of such steam exploded wood pulps.

Another possibility for explaining the difficulty to dissolve cotton fibres and wood pulps is that the insoluble areas would contain non-cellulosic chains and products. This is not a general rule since insoluble parts may contain a larger fraction of cellulose (measured by the glucose content) than the original product (Isogai and Atalla 1998).

Aside from the facts that some parts of pulp fibres are not dissolving well, it is interesting to look in a closer way at the areas in the fibre that may have different dissolving capacity. As already said, it was shown for example that in the case of cotton fibres, the younger cellulose chains are easier to dissolve. In the present work, we dissolved five different cellulose samples in a bad solvent $(\mathrm{NaOH}-8 \%$ water), then separated, analysed and compared the insoluble and clear solution fractions. The resulting fractions were observed by optical microscopy and transmission electron microscopy. Molecular weight distribution, carbohydrate composition and cellulose II content were measured. The aim of this study was to better understand the dissolution mechanisms of wood fibres in $\mathrm{NaOH}$-water systems and to better identify and characterize the regions in the fibres that have different dissolution capacities. 


\section{Experimental Part}

\section{Sample and solution preparation}

Five samples were studied: two dissolving wood pulps prepared by steam explosion and provided by Borregaard (Norway), a microcrystalline cellulose (MCC) sold by Sigma-Aldrich, a bleached sulphite pulp provided by Borregaard and a PH Kraft pulp provided by the vTI-Institute of Wood Technology and Wood Biology (Hamburg, Germany). The origin of the cellulose, the type of pretreatment, the degree of polymerization (DP) given by the providers and the fibre / particle diameter are reported in Table 1.

The different samples were dissolved in a $\mathrm{NaOH} 8 \%$ - water solution at the concentration of $1 \%$ cellulose by weight. The cellulose solutions were prepared as follows: 330 grams of $\mathrm{NaOH} 12 \%$ - water were stored in a freezer. Five grams of cellulose sample were dried at $50^{\circ} \mathrm{C}$ overnight then added to 165 grams of distilled water and stored 1 hour at $4{ }^{\circ} \mathrm{C}$. Then, $\mathrm{NaOH} 12 \%$ - water and cellulose water solutions were mixed together using a rotary overhead mixer during two hours at $-6^{\circ} \mathrm{C}$ and $1000 \mathrm{rpm}$ giving 500 grams of a solution of $1 \%$ cellulose in $\mathrm{NaOH} 8 \%$ - water. A supplementary solution of the SE DP 403 pulp was prepared in the same conditions without the mixing at $1000 \mathrm{rpm}$ with only a gentle stirring applied at the beginning of the preparation to ensure a good dispersion of the fibres in the solution. Three solutions were prepared for each sample to check reproducibility of the experiments and measurements. These $1 \%$ cellulose solutions were centrifuged without further treatment by following the experimental protocol shown in Figure 1 and described in the next paragraph.

\section{Fractionation by centrifugation}

Centrifugation was performed on a Hettich Universal 320RHK centrifuge equipped with a 1620A rotor and a Julabo cryostat. By increasing the centrifugation velocity, it was possible to isolate different fractions from the solutions (Figure 1). Protocols were adjusted following a preliminary study, not reported here. For the SE DP 403 and SE DP 360 samples, a protocol in three steps was tailored to separate the large, medium and small insoluble parts (insoluble fractions $I_{1}, I_{2}$ and $I_{3}$ respectively) and the clear solution fraction $(S)$. The centrifuge tubes were refrigerated at $4^{\circ} \mathrm{C}$ before starting centrifugation. Three 
rotation speeds were successively applied: $2250 \mathrm{rpm}(560 \mathrm{~g}), 4750 \mathrm{rpm}(2520 \mathrm{~g})$ and finally $9000 \mathrm{rpm}(9050 \mathrm{~g})$. Despite the cryostat of the centrifuge was set at $0^{\circ} \mathrm{C}$, the temperature inside the rotating sample increased up to 8 and $12^{\circ} \mathrm{C}$ due to centrifugation heating and heat loss between the cryostat and the centrifuge. The centrifugation time for each step was 5 minutes. The low concentration of cellulose $(1 \%)$, the low temperature (below $12^{\circ} \mathrm{C}$ ) and the short centrifugation time (5 min) used prevent the gelation of the solutions during centrifugation (Roy et al. 2003). As will be discussed in the Results section, the clear solution fraction is translucent at a microscopic scale but it should be composed of aggregated cellulose chains and very small submicron cellulose fragments.

In the case of Avicel PH 101, bleached sulphite pulp, PH Kraft pulp and nonagitated SE DP 403 samples, only one centrifugation step at maximum speed 9000 rpm (9050 g) was applied and two fractions were obtained: an insoluble fraction (I) and a clear solution fraction (S).

\section{Optical and transmission electron microscopy observations}

After the centrifugation protocol, all fractions were observed by optical microscopy between two glass plates in transmission mode with and without cross-polarizers with a Metallux 3 (Leitz) equipped with a high resolution 3-CCD camera JVC KY-F75U.

The clear solution fraction (S) was also observed by Transmission Electron Microscopy (TEM) with a CM12 TEM/STEM Philips. A droplet was deposited on a copper grid covered with a Formvar resin and a carbon film. The grid was deposited on a blotting paper to remove the solvent and then on the TEM sample holder. The accelerating voltage was adjusted at $120 \mathrm{KV}$ and the magnification was 8800 .

\section{Precipitation and weighing of the fractions}

The different fractions were precipitated with distilled water in several washing baths until the $\mathrm{pH}$ was neutral. As will be discussed in the Results section, a part of the materials present in the clear solution fractions was not recovered after precipitation and neutralization. After precipitation, the clear solution fraction (S) was thus divided in two fractions the recovered fraction $\left(S_{r}\right)$ and the nonrecovered fraction $\left(S_{n r}\right)$. The precipitated products, i.e. fractions $\left(I_{1}, I_{2}, I_{3}\right.$ and $\left.I\right)$ 
and fraction $\left(\mathrm{S}_{\mathrm{r}}\right)$, were dried overnight in an air oven at $50^{\circ} \mathrm{C}$ and were weighed after total drying. The amount of each fraction was defined as the weight ratio [dried fraction] / [dried sample introduced in the solution]. The amount of nonrecovered fraction $\left(S_{n r}\right)$ was deducted from the amount of recovered fraction $\left(S_{r}\right)$ and insoluble fraction.

\section{Molecular weight distribution, carbohydrate composition and cellulose II content}

The molecular weight distribution of each insoluble fraction $\left(\mathrm{I}_{1}, \mathrm{I}_{2}, \mathrm{I}_{3}\right.$ and $\left.\mathrm{I}\right)$ and recovered fraction $\left(\mathrm{S}_{\mathrm{r}}\right)$ was investigated by underivatized size exclusion chromatography in DMAc / $\mathrm{LiCl}$ with refractive index detection coupled with multi angle laser light scattering. Their carbohydrate composition was determined by HPLC (anion chromatography with pulsed amperometric detection) after a two steps sulfuric acid hydrolysis. The carbohydrate composition of the non-recovered fraction $\left(\mathrm{S}_{\mathrm{nr}}\right)$ was deducted from that of the insoluble and the recovered fractions $\left(\mathrm{I}_{1}, \mathrm{I}_{2}, \mathrm{I}_{3}, \mathrm{I}\right.$ and $\left.\mathrm{S}_{\mathrm{r}}\right)$. The cellulose II content was evaluated by a FTIR-ATR comparative method calibrated by wide angle X-ray scattering and by ${ }^{13} \mathrm{C}$ NMR (Baldinger et al. 2000). The evaluation and correlation of IR-spectra from various samples fully characterized by wide angle X-ray scattering and ${ }^{13} \mathrm{C}$ NMR give information on type of crystal lattice (cellulose I, II ...). The results are given with an uncertainty of $\pm 5 \%$. All the analyses were performed by Lenzing $A G$ (Austria).

\section{Results}

\section{Steam exploded (SE) wood pulps: observations of fraction $I_{1}$, large insoluble parts (low rotation speed, $560 \mathrm{~g}$ )}

The optical microscopy observations of fraction $I_{1}$ of wood pulps reveal only very few completely undissolved fibres. Some of these fibres are as the original fibres, but a few are swollen and shows a ballooning with diameters around 50$100 \mu \mathrm{m}$ with unswollen sections and thick helices (Figure 2). The ballooning phenomenon is due to the swelling and dissolution of the secondary wall which induce the breaking of the primary wall, forming unswollen sections and thick helices (Le Moigne et al. 2008). The observation of some ballooned fibres in 
fraction $I_{1}$ indicates that the external walls, especially the primary wall, are still present on these fibres. However, most of the fibres seen in fraction $I_{1}$ are highly and homogeneously swollen (230-560\% of swelling) with diameters of about 100 $\mu \mathrm{m}$ and their primary wall is not present anymore, i.e. no unswollen sections and helices are observed. Only the secondary wall, including S1 wall and S2 wall, is present in these fibres inducing a regular swelling all along the fibres. These observations show that the steam explosion pre-treatment of the pulps leads to various effects on the fibres, some fibres keeping their primary wall nearly intact, some loosing it but having their secondary walls not touched, while most of the fibres were destructured enough to loose their integrity during treatment with the solvent. These differences in efficiency of the steam explosion may be due to heterogeneities from one fibre to another in the same sample (variation of sulphite treatment efficiency, type of cells...). As an example, softwood pulps are more sensitive to steam explosion than hardwood pulps (Yamashiki et al. 1990a).

Sections of several hundred microns length, $100 \mu \mathrm{m}$ diameters and numerous flat rings with external diameters of $100 \mu \mathrm{m}$, internal diameters of about 10 to 50 $\mu \mathrm{m}$ are also observed (Figure 2). Similar sections and flat rings were first described by Stawitz and Kage (1959) after the swelling of carboxymethyl cellulose (CMC). More recently, undissolved residuals were observed by optical microscopy in CMC solutions and it was suggested that highly swollen sections and flat rings may come from the ballooned fibres or from swelled pores (Jardeby et al. 2005). Considering the dimension and the shape of the sections and the rings, the hypothesis that they come from the cutting of the highly swollen fibres is the most probable. We will come to this point later.

\section{Steam exploded (SE) wood pulps: observations of fraction $I_{2}$, medium insoluble parts (higher centrifugation speed, $2520 \mathrm{~g}$ )}

This fraction does not show any large pieces of undissolved fibres as in fraction $\mathrm{I}_{1}$. Only numerous flat rings were observed with diameters equivalent to those observed in fraction $I_{1}$. Lots of fragments with various sizes and shapes from 50 to $100 \mu \mathrm{m}$ were also observed. Looking at their shapes, we can deduce that these fragments come from the breaking of flat rings due to the shear stresses occurring during mixing (Figure 3). As can be seen, some fragments show a 
circular shape reminding those of the rings. However, it is also possible that fibres tear directly into fragments without going through the stage of flat rings.

\section{Steam exploded (SE) wood pulps: observations of fraction $I_{3}$, small insoluble parts (high centrifugation speed, $9050 \mathrm{~g}$ )}

After this last centrifugation stage, small fragments of about $10-50 \mu \mathrm{m}$ were collected (Figure 4). These little fragments are coming from the breaking of the fibres, the flat rings and their large fragments. The mixing of the solution during preparation decreases the size of insoluble parts due to shear stresses induced and the resulting solution is clearer. However, many small insoluble parts are still present in the solution showing that the breaking of the fibres in small pieces is not the only parameter governing the dissolution of wood fibres in $\mathrm{NaOH}$-water systems.

\section{Steam exploded (SE) wood pulps: observations of fraction S, clear solution fraction}

This last fraction is an optically clear solution of cellulose. Some very thin insoluble parts with their largest dimension being smaller than $3 \mu \mathrm{m}$ were observed by TEM (Figure 5) showing that the fractionation by centrifugation was not fully effective for removing all undissolved materials from the supernatant. This should slightly decrease the differences between the insoluble and the clear solution fractions. It has also to be noticed that cellulose chains are always in an aggregated state even in very good solvent such as $N$-methylmorpholine- $N$-oxyde - monohydrate (Schulz et al. 2000). The clear solution fraction should thus be composed of aggregated cellulose chains and very small submicron fragments.

\section{Steam exploded (SE) wood pulps: influence of the mixing on the shapes of insoluble parts}

All the above reported results were obtained with solution sheared at $1000 \mathrm{rpm}$ for two hours in a mechanical mixer. The influence of this mixing process was tested by preparing a solution of SE DP 403 with the same preparation protocol, but without agitation. The resulting solution was then observed with the same tools. As can be seen on Figure 6, highly swollen sections and flat rings are also present for this solution preparation without mechanical mixing. These flat rings 
are thus not due to the shearing in the mixer but the swelling itself is sufficient to dismantle the fibre in sections and rings. The shearing involved by the agitation is thus not the main parameter that governed the dismantlement in sections and flat rings. However, the shearing is necessary for the breaking of the large flat rings in smaller and smaller fragments.

\section{Wood pulps: Observations of the insoluble fraction I for PH Kraft pulp and bleached sulphite pulp}

The effect of the cellulose chemical pre-treatments has been tested by studying the morphology of insoluble parts in other wood fibres, i.e. a bleached sulphite pulp and a PH Kraft pulp. In the case of the PH Kraft pulp solution, most of the fibres are only slightly swollen. However, as can be seen on Figure 7, highly swollen fibres, sections and flat rings are observable in both samples. It means that these flat rings are not due the steam explosion pre-treatment or differences of chemical action between the sulphate and the sulphite cooking. It has to be noticed that the flat rings show a lower swelling and present a rectangular shape in the case of the PH Kraft pulp sample which reminds the original shape of a non swollen wood fibre. This has to be related with the presence of the external walls, especially the primary wall, which prevents swelling in such bad solvent.

\section{Microcrystalline cellulose: observations of the insoluble fraction I}

Avicel $\mathrm{PH} 101$ (DP 170) is not fully dissolving in $\mathrm{NaOH} 8 \%$ - water. Centrifugation clearly produced a very small fraction containing insoluble parts. These insoluble parts, having their largest dimension of about 1 to $10 \mu \mathrm{m}$, can be observed even for a 1\% Avicel PH 101 solution (Figure 8) and are fragment-like pieces as those observed in fraction $I_{3}$ from steam exploded wood pulp samples. Observations between cross-polarizer indicate that cellulose chains are oriented in these insoluble fragments.

\section{Weighing of the fractions}

After centrifugation, precipitation in distilled water and drying at $50^{\circ} \mathrm{C}$ overnight, the weight of the solid material of each fraction, i.e. $I_{1}, I_{2}, I_{3}$, I and $S_{r}$, was measured. The detailed weight fractions are reported in Table 2 for SE DP 360, SE DP 403 samples and Avicel PH 101 (each value is an average of three 
measurements). About 28-30\% of the total product is non-recovered. This lost product is not coming from the insoluble fractions which are giving highly viscous and sticky centrifugation pellets easy to recover. It is mainly coming from the clear solution fraction where cellulose is solubilized in a large amount of solvent and then precipitated and neutralized in very large amounts of water with at least five washing baths. Some materials and low molar mass products are thus washed out upon precipitation and neutralization and this fraction is called nonrecovered fraction $\left(\mathrm{S}_{\mathrm{nr}}\right)$.

As seen in Figure 9, the PH Kraft pulp is not soluble in $\mathrm{NaOH} 8 \%$ - water. The amount of insoluble material for the PH Kraft pulp sample is very high (around $80 \%$ ) due its high DP and mainly to the fact that fibres have not been too much destructured during the sulphate pre-treatment. The bleached sulphite treatment leads to a medium quantity of insoluble parts $(42 \%)$ due to the decrease of DP, which was shown to be linearly correlated with the alkaline solubility (Yamashiki et al. 1990b), and to the loosening of integrity of the original wall structure. Without agitation, the quantity of insoluble material for the SE DP 403 sample is increased by three times (38\% vs $12 \%$ for agitated solutions) stressing the role of the shearing in the dissolution efficiency. The lowest insoluble material amount is reached with the Avicel PH 101 (2-4 \%) sample which is the most destructured sample.

\section{Molecular weight distributions of the fractions}

The molecular weight distributions of the insoluble fractions $\left(I_{1}, I_{2}, I_{3}, I\right)$ have higher mean molar masses than the mean molar masses of the original samples (Figure 10). For the two steam exploded samples, SE DP 360 and SE DP 403, the average molecular weights $\mathrm{Mw}$ of the insoluble fractions are 1.5 - 2 times higher than the one of the recovered fractions $\left(S_{\mathrm{r}}\right)$, i.e. $120000-160000$ g.mol ${ }^{-1}$ versus $76000 \mathrm{~g} \cdot \mathrm{mol}^{-1}$. The average molecular weights $\mathrm{Mn}$ of insoluble fractions are also roughly 1.5 times higher than the recovered fractions $\left(S_{r}\right)$, i.e. $59000-65000$ g.mol ${ }^{-1}$ versus $39000-43000$ g.mol ${ }^{-1}$.

Molecular weight distributions of the insoluble fractions and recovered fractions $\left(\mathrm{S}_{\mathrm{r}}\right)$ of the Avicel PH 101 are similar. As previously discussed, the insoluble fraction is made of fragments ( $2-4 \%$ by weight) that have relatively low average Mn (35 000 g. $\mathrm{mol}^{-1}$ ). These fragments remain insoluble although the original wall 
structure is supposed to be strongly disrupted by the acidic hydrolysis pretreatment. The fine structure and the composition at the molecular level should thus strongly influence the dissolution capacity.

The polymolecularity index is higher for the insoluble fractions than for the recovered fractions $\left(\mathrm{S}_{\mathrm{r}}\right)$, i.e. $\approx 2.35$ and 1.8 respectively for SE DP 403 and SE DP 360 wood pulps, and 1.9 and 1.6 respectively for Avicel PH 101. Insoluble fractions contain small chains as in the original samples and the recovered fractions $\left(\mathrm{S}_{\mathrm{r}}\right)$ together with a large share of long chains. The fact that some cellulose chains did not dissolve while some others did, although having the same molecular weight, indicates that these chains were less accessible and embedded in regions difficult to dissolve. It is not simply long chains that are not dissolving but also complex regions where shorter chains can be present. Beyond thermodynamic considerations, it shows that the dissolution capacity of cellulose chains is very dependant of their localization in the cell wall structure. The length of the chains is indeed not the main parameter, since otherwise molecular weight distributions of the insoluble fractions and the recovered fractions $\left(\mathrm{S}_{\mathrm{r}}\right)$ would be clearly different. Considering the large superposition of the molar mass distributions of the insoluble fractions and the recovered fractions $\left(\mathrm{S}_{\mathrm{r}}\right)$, the fact that a part of the clear solution fractions has not been recovered should not change the above conclusions.

\section{Carbohydrate composition of the fractions}

The carbohydrate composition of the different fractions from the two steam exploded wood pulps shows that a large part of the hemicelluloses, i.e. xylans and mannans (90\% and $72 \%$ respectively), are contained in the non-recovered fractions $\left(\mathrm{S}_{\mathrm{nr}}\right)$ meaning that the hemicelluloses were solubilized in $\mathrm{NaOH} 8 \%$ water and washed out upon precipitation and neutralization of the cellulose (Table 3). A part of the glucans $(27-28 \%)$ is also contained in the non-recovered fractions $\left(\mathrm{S}_{\mathrm{nr}}\right)$. However, a part of the hemicelluloses $(10 \%$ of xylans and $28 \%$ of mannans) stays aggregated with the cellulose in both insoluble fractions and recovered fractions $\left(\mathrm{S}_{\mathrm{r}}\right)$. In the case of the recovered fractions $\left(\mathrm{S}_{\mathrm{r}}\right)$, the presence of hemicelluloses should come from their trapping during the regeneration in distilled water. It has to be noticed that the ratios mannan / xylan and glucan / mannan are significantly different for the insoluble fractions $\left(I_{1}+I_{2}+I_{3}\right)$ as 
compared to the recovered fractions $\left(\mathrm{S}_{\mathrm{r}}\right)(2.44-3.60$ vs 1.5 and $165-174$ vs 332 respectively). It is also interesting to note that hemicelluloses are present in the Avicel PH 101 sample even after the acidic hydrolysis pre-treatment and that the same is observed concerning the ratios mannan / xylan and glucan / mannan (3.92 vs 1 and 126 vs 496 respectively). These results show that mannans are more strongly associated with the cellulose than xylans and that higher concentration of mannans are present in the insoluble fractions.

The strong interactions between cellulose and hemicelluloses have been illustrated by several works. As was reported by Isogaï and Atalla (1998), these interactions are supposed to be physical, in the sense of co-aggregation (Hackney et al. 1994) or based on chemical linkages (Isogaï et al. 1989). It has been also suggested that glucomannans are more associated with cellulose while xylans are more associated with lignin (Iwata et al. 1998; Salmén and Olsson 1998). These results were supported by dynamic FT-IR spectroscopy investigations (Åkerholm and Salmén 2001). Isogai and Atalla (1998) assume that the presence of hemicelluloses does not seem to prevent dissolution because most of hemicelluloses fractions are solubilize in aqueous $\mathrm{NaOH}$. However, the authors only compared hemicelluloses content in original samples and insoluble fractions with no measurements done on the soluble ones. Our results show that there is a significant amount of mannans in the insoluble fractions. In comparison, the amount of mannans that is trapped in the recovered fraction $\left(\mathrm{S}_{\mathrm{r}}\right)$ is almost twice less when considering the ratios mannan / xylan and glucan / mannan. Mannans in the insoluble fractions are not dissolved in the solution and are attached to the cellulose chains contrary to the chains which will undergo dissolution. The way cellulose and hemicelluloses are linked and probably organized (Fahlén 2005; Fengel 1971; O'Sullivan 1997) should play an important role upon the dissolution of cellulose.

The variations in dissolution capacity of cellulose chains shown by the above molecular weight distribution results might be related to the localization of the cellulose chains in the cellulose / hemicelluloses complex, i.e. cellulose chains are more or less embedded in a hemicelluloses matrix, thus influencing the dissolution efficiency. Why some hemicelluloses are non-soluble and stay aggregated with the cellulose while a large part of it solubilizes easily in $\mathrm{NaOH}$ $8 \%$ - water is an interesting question. There should be also variations of 
accessibility and solubilization of the hemicelluloses related to their localization within the cell wall structure.

\section{Cellulose II content of the fractions}

The cellulose II content in insoluble fractions and recovered fractions $\left(\mathrm{S}_{\mathrm{r}}\right)$ was investigated by FTIR-ATR using a comparative method (Baldinger et al. 2000). The deconvoluted $\mathrm{OH}$ regions (bands around 2720, 3278, 3344, 3422, 3450 and $3700 \mathrm{~cm}^{-1}$ with $2900 \mathrm{~cm}^{-1}$ as reference band) of the samples spectra were compared with those of several cellulose I / cellulose II composition references prepared in $\mathrm{NaOH}$ - water at various concentration (Figure 11). The band 2900

$\mathrm{cm}^{-1}$, assign to $\mathrm{CH}_{2}$ stretching, is commonly used as reference band because it is unaffected by changes in sample preparation, crystallinity or humidity and shows a constant intensity (Nelson and O'Connor 1964). The cellulose I / cellulose II composition values of the references were calibrated by ${ }^{13} \mathrm{C} N \mathrm{NR}$. The $\mathrm{OH}$ region area is larger for cellulose II than cellulose I and thus increases with the amount of cellulose I converted in cellulose II. The content of cellulose II is deducted from the calculated area of the $\mathrm{OH}$ region which is specific to a cellulose I / cellulose II composition. The results are given with an uncertainty of $\pm 5 \%$.

The results presented in Table 4 shows that all the cellulose I was converted in cellulose II even for insoluble fractions. The crystal structure of highly swollen fibres, sections and flat rings after regeneration is thus cellulose II. Even for the cellulose chains present in the insoluble fractions, the large swelling gives enough molecular mobility to convert cellulose I in cellulose II, as it is doing in the mercerisation process.

\section{Discussion}

The dissolution of wood fibres in $\mathrm{NaOH}$ - water is not very efficient. Aside thermodynamic limitations due to the $\mathrm{NaOH} / \mathrm{AGU}$ ratio needed to solubilize cellulose (Egal et al. 2007) and the fast gelation of the solutions (Roy et al. 2003), the results reported here show that dissolution proceeds in a complex way that strongly depends on the location of the cellulose chains inside the cell structure. We already showed that there is a gradient of dissolution capacity inside the cell wall structure (Le Moigne et al. 2008). The younger deposited layers are easier to dissolve than the oldest ones; i.e. cellulose embedded into the primary wall or into 
the secondary S1 wall close to the primary wall is more difficult to dissolve than the one deep inside the secondary S2 wall, close to the cell nucleus.

The results reported here show that the dissolution picture is even more complicated. The dissolution capacity of wood fibres must be considered at two levels, (i) the macrostructure that needs to be dismantled for increasing dissolution, and (ii) the cellulose chain chemical environment.

\section{Macrostructural level}

The destructuration of the layer structure by steam explosion, acidic hydrolysis or agitation helps the dissolution. As for all polymer dissolution, agitation is removing concentrated solution away from the dissolving part and brings fresh solvent. With agitation, the quantity of insoluble material is decreased by three showing the role of agitation on the dissolution process (Figure 9). This is facilitated in wood pulps by treatments like steam explosion as was demonstrated earlier by Yamashiki et al. (1990a, b, c). Our results show that the steam explosion of the SE DP 360 and SE DP 403 samples acts as a destructuration treatment which facilitates the dismantlement and the fragmentation of the fibres in the $\mathrm{NaOH} 8 \%$ - water mixture and finally decreases the insoluble material amount (10 - $14 \%$ vs $42 \%$ for the bleached sulphite pulp).

\section{Cellulose chain chemical environment}

The fact that short cellulose chains did not dissolve while some longer one did, indicates that some chains are less accessible and embedded in regions difficult to dissolve. It shows that the dissolution capacity of cellulose chains, beyond thermodynamic considerations, is very dependant of their localization in the cell wall structure. The higher concentration of hemicelluloses, especially mannans, in the insoluble fractions suggest that the variations in dissolution capacity of cellulose chains might be related to the localization of the cellulose chains in the cellulose / hemicelluloses complex, i.e. cellulose chains are more or less embedded in a hemicelluloses matrix.

\section{Origin of the flat rings}

The reasons that lead to the particular "flat rings" geometry need to be more detailed. To what extent the cutting in flat rings is due to the original structure of 
the fibre, to the chemical or physical pre-treatment or to the swelling and dissolution undergone by the fibres is an interesting question?

Dissolution produces different parts that correspond to successive morphologies during dissolution: the insoluble fraction containing the highly swollen fibres, the highly swollen sections, the flat rings, the fragments and the clear solution fraction. This mechanism of dismantlement and fragmentation is mainly governed by the high swelling and in a lower extent by the shear stress involved during the solution preparation. The different steps of dissolution of wood cellulose fibres in $\mathrm{NaOH} 8 \%$ - water are schematically represented on Figure 12.

In the highly swollen fibres, we have shown previously that the primary wall is not present due to the absence of ballooning. Only the S1 wall and the S2 wall are thus present in these highly swollen fibres. Cellulose microfibrils in wood fibre generally have orientation angle of about $50-70^{\circ}$ (Roelofsen 1959) or $70-90^{\circ}$ (Brändström et al. 2003) in S1 wall and 0-30 in S2 wall (Sahlberg et al. 1997). The large swelling of the fibre involves a regular tear of the outer layer, i.e. the S1 wall, every $10 \mu \mathrm{m}$ which is clearly visible on Figure 2. Numerous tears transverse to the fibre axis (white lines) are observable along highly swollen fibres and sections. Considering the high radial swelling (230 to $560 \%$ ), the outer S1 wall must tear because its microfibrils are almost perpendicular to the fibre axis. The regular repetition of these tear every $10 \mu \mathrm{m}$ still have to be explained but it has to be related with the deposition and the cohesion of the cellulose microfibrils in the $\mathrm{S} 1$ wall. The tearing zones can be considered as weak points where the fibre can break in sections and flat rings. This mechanism requires that the S2 wall is transversely cut while its microfibrils are almost parallel $\left(0-30^{\circ}\right)$ to the fibre axis. The loss of mechanical properties of the $\mathrm{S} 2$ wall can be explained by the fact that the original compact cellulose structure is not preserved since the S2 wall is highly swollen (230-560\%) and partially dissolved. A shear stress is thus not essential to cut the highly swollen fibres in sections and flat rings. It has to be noticed that the cutting of the highly swollen fibres in rings and not in disks shows that the wood fibres were not fully mature or that the inside tertiary wall and wart layer were removed by the pre-treatments or easily soluble in the $\mathrm{NaOH} 8 \%$ water solution.

The flat rings observed with PH Kraft pulp show that a high degree of swelling is not necessary and, in this case, we must assume that shearing was the main 
factor of the cutting in flat rings. All these experiments show that wood fibres can dismantle in flat rings when putting in $\mathrm{NaOH} 8 \%$ - water, independent of their original cooking (steam explosion pre-treatment, sulphite or sulphate processes).

\section{Limit of solubility}

The limit of solubility of cellulose in $\mathrm{NaOH} 8 \%$ - water at $-6^{\circ} \mathrm{C}$ in terms of DP can be approximated as the average DPn and DPw of the recovered fractions $\left(\mathrm{S}_{\mathrm{r}}\right)$, $250\left(\mathrm{Mn} / 162 \mathrm{~g} \cdot \mathrm{mol}^{-1}\right)$ and $470\left(\mathrm{Mw} / 162 \mathrm{~g} \cdot \mathrm{mol}^{-1}\right)$ respectively. These values have to be considered as an average and the molecular weight distributions shows that longer chains are also dissolved. Yamashiki et al. (1990a) assumed that the alkaline solubility of cellulose samples can be improve up to $100 \%$ when the DPw value is decrease below 400 after steam explosion. Maximum values of DPn (107) and DPw (268) were recently reported for enzymatically treated samples dissolved and filtrated in 7.8 wt. $\% \mathrm{NaOH}$ and 0.84 wt. $\% \mathrm{ZnO}$ at $3^{\circ} \mathrm{C}$, then spun into $15 \%$ sulphuric acid (Vehviläinen et al. 2008). All these data are not coming from pure cellulose sample, but from wood pulps and cotton linters. In such samples, cellulose chains are embedded in a complex and compact layer structure. As an example, in 8.5 wt. $\% \mathrm{NaOH}$ and 1 wt. $\%$ urea at $-5^{\circ} \mathrm{C}$, bacterial cellulose was shown to be dissolved so long as its DPw is below 560 (Łaszkiewicz 1998), stressing the adverse influence of the cell wall structure and composition on the dissolution capacity.

\section{Conclusions}

It is often reported that cellulose must be accessible for being dissolved or chemically treated. Our results show that the molecular weight cannot be considered as the main factor in the cellulose dissolution. The macrostructure and the chemical environment of the cellulose chains are of primary importance. The presence of small amounts of hemicelluloses may prevent or decrease the solubility of cellulose. The peculiar splitting of the fibres into rings is clearly due to the morphology of the fibres. The fact that these flat rings have a rather similar thickness suggest that there is a similar periodical heterogeneity along the fibres. It must also be kept in mind that what is called "cellulose solution" is surely not a molecular solution in the thermodynamic sense, and that cellulose aggregates are mainly present. 
Acknowledgements. The authors thank J. Engelhardt, K. Jardeby, T. Hjerde, H. Harms, M. Pierre and C. Schrempf for discussions and Borregaard, Dow Wolff Cellulosics GmbH, Lenzing AG and Spontex for their technical and financial support. We also thank B Saake and J. Puls from the vTIInstitute of Wood Technology and Wood Biology for discussions and providing the kraft sample and the Institut of Molecular and Cellular pharmacology (IPMC) for their help in centrifugation.

\section{References}

Åkerholm M, Salmén L (2001) Interactions between wood polymers studied by dynamic FT-IR spectroscopy. Polymer 42:963-969

Baldinger T, Moosbauer J, Sixta H (2000) Supermolecular structure of cellulosic materials by Fourier transform infrared spectroscopy (FT-IR) calibrated by WAXS and ${ }^{13} \mathrm{C}$ NMR. Lenzinger Ber 79:15-17

Brändström J, Bardage SL, Daniel G, Nilsson T (2003) The structural organisation of the $S_{1}$ cell wall layer of Norway spruce tracheids. IAWA J. 24:27-40

Chanzy H, Noe P, Paillet M, Smith P (1983) Swelling and dissolution of cellulose in amine oxide / water systems. J Appl Polym Sci 37:239-259

Cuissinat C (2006) Swelling and dissolution mechanisms of native cellulose fibres. $\mathrm{PhD}$ dissertation, Ecole Nationale Supérieure des Mines de Paris, Sophia-Antipolis, France

Cuissinat C, Navard P (2006a) Swelling and dissolution of cellulose, Part I: free floating cotton and wood fibres in $N$-methylmorpholine- $N$-oxide-water mixtures. Macromol Symp 244:1-15

Cuissinat C, Navard P (2006b) Swelling and dissolution of cellulose, Part II: free floating cotton and wood fibres in $\mathrm{NaOH}$ water-additives systems. Macromol Symp 244:19-30

Cuissinat C, Navard P (2008) Swelling and dissolution of cellulose, Part III: Plant fibres in aqueous systems. Cellulose 15:67-74

Cuissinat C, Navard P, Heinze, T (2008a) Swelling and dissolution of cellulose, Part IV: Free floating cotton and wood fibres in ionic liquids. Carbohydr Polym 72:590-596

Cuissinat C, Navard P, Heinze T (2008b) Swelling and dissolution of cellulose, Part V: Cellulose derivatives fibres in aqueous systems and ionic liquids. Cellulose 15:75-80

Egal M, Budtova T, Navard P (2007) Structure of aqueous solutions of microcrystalline cellulosesodium hydroxide below $0^{\circ} \mathrm{C}$ and the limit of cellulose dissolution. Biomacromolecules $8: 2282-2287$

Fahlén J (2005) The cell wall ultrastructure of wood fibers: Effects of the chemical pulp fiber line. PhD dissertation, KTH Royal Institute of Technology, Stockholm, Sueden

Fengel D (1971) Ideas on ultrastructural organisation of cell-wall components. J Polym Sci: part C 9:383-392

Flemming N, Thaysen AC (1919) On the deterioration of cotton on wet storage. Biochem J $14(1): 25-28$ 
Hackney JM, Atalla RH, VanderHart DL (1994) Modification of crystallinity and crystalline structure of Acetobacter xylinum cellulose in the presence of water-soluble beta-1,4-linked polysaccharides: ${ }^{13} \mathrm{C}$ NMR evidence. Int J Biol Macromol 16:215-218

Hock CW (1950) Degradation of cellulose as revealed microscopically. Text Res J 20:141-151

Hock CW (1954) Microscopic structure. In: Ott E, Spurlin HM, Grafflin MW (eds) Cellulose and cellulose derivatives (Part 1), 2nd edn. Interscience Publisher, New York, London, pp 347-392

Isogai A, Ishizu A, Nakano J (1989) Residual lignin and hemicellulose in wood cellulose, analysis using new permethylation method. Holzforschung 43:333-338

Isogai A, Atalla RH (1998) Dissolution of cellulose in aqueous NaOH solutions. Cellulose 5:309319

Iwata T, Indrarti L, Azuma JI (1998) Affinity of hemicellulose for cellulose produced by Acetobacter Xylinum. Cellulose 5:215-228

Jardeby K, Germgård U, Kreutz B, Heinze T, Heinze U, Lennholm H (2005) The influence of fibre wall thickness on the undissolved residuals in CMC solutions. Cellulose 12:167-175

Łaszkiewicz B (1998) Solubility of bacterial cellulose and its structural properties. J Appl Polym Sci 67:1871-1876

Le Moigne N, Montes E, Pannetier C, Höfte H, Navard P (2008) Gradient in Dissolution Capacity of Successively Deposited Cell Wall Layers in Cotton Fibres. Macromol Symp 262:65-71

Le Moigne N (2008) Swelling and dissolution mechanisms of cellulose fibres. PhD dissertation, Ecole Nationale Supérieure des Mines de Paris, Sophia Antipolis, France

Nägeli C (1864) Über den inneren Bau der vegetabilischen Zellmembranen. Sitzber Bay Akad Wiss München 1:282-323, 2:114-171

Nelson ML, O’Connor RT (1964) Relation of certain infrared bands to cellulose crystallinity and crystal lattice type. Part II. A new infrared ratio for estimation of crystallinity in celluloses I and II. J Appl Polym Sci 8:1325-1341

O’Sullivan AC (1997) Cellulose: the structure slowly unravels. Cellulose 4:173-207

Pennetier G (1883) Note micrographique sur les altérations du cotton. Bull Soc Ind Rouen 11:235237

Roelofsen PA (1959) The cell-wall structure. In: The plant cell-wall. Gebrüder Borntraeger, Berlin, pp 102-305

Rollins ML, Tripp VW (1954) Optical and electron microscopic studies of cotton fiber structure. Text Res J 24:345-357

Roy C, Budtova T, Navard P (2003) Rheological properties and gelation of aqueous cellulose$\mathrm{NaOH}$ solutions. Biomacromolecules 4:259-264

Sahlberg U, Salmén L, Oscarsson A (1997) The fibrillar orientation in the S2 layer of wood fibres as determined by X-ray diffraction analysis. Wood Sci Technol 31:77-86

Salmén L, Olsson AM (1998) Interaction between hemicelluloses, lignin and cellulose: structureproperty relationships. J Pulp Pap Sci 24:99-103

Schulz L, Seger B, Burchard W (2000) Structures of cellulose in solution. Macromol Chem Phys 201:2008-2022 
Stawitz J, Kage MP (1959) Über die Quellungsstadien der wasserlöslichen Celluloseäther und die übermolekulare Struktur der Celllose. Das Papier 13:567-572

Tripp VW, Rollins ML (1952) Morphology and chemical composition of certain components of cotton fiber cell wall. Anal Chem 24:1721-1728

Vehviläinen M, Kamppuri T, Rom M, Jaroslaw J, Ciechańska D, Grönqvist S, Siika-Aho M, Christoffersson KE, Nousiainen P (2008) Effect of wet spinning parameters on the properties of novel cellulosic fibres. Cellulose 15:671-680

Yamashiki T, Matsui T, Saitoh M, Okajima K, Kamide K, Sawada T (1990a) Characterization of cellulose treated by the steam explosion method. Part 1. Influence of cellulose resources on changes in morphology, degree of polymerization, solubility and solid structure. Br Polym J 22:73-83

Yamashiki T, Matsui T, Saitoh M, Okajima K, Kamide K, Sawada T (1990b) Characterization of cellulose treated by the steam explosion method. Part 2: Effect of treatment conditions on changes in morphology, degree of polymerization, solubility in aqueous sodium hydroxide, and supermolecular structure of soft wood pulp during steam explosion. Br Polym J 22:121-128

Yamashiki T, Matsui T, Saitoh M, Okajima K, Kamide K, Sawada T (1990c) Characterization of cellulose treated by the steam explosion method. Part 3: Effect of crystal forms (cellulose I, II and III) of original cellulose on changes in morphology, degree of polymerization, solubility and supermolecular structure by steam explosion. Br Polym J 22:201-212 


\section{Table legends}

Table 1 Properties of the wood-based cellulose samples used for fractionation by centrifugation.

Table 2 Amount of fractions $I_{1}, I_{2}, I_{3}, I, S_{r}$ and $S_{n r}$ after centrifugation, precipitation and drying for the SE DP 360 and SE DP 403 pulps and Avicel PH 101.

Table 3 Carbohydrate compositions of original samples, insoluble fractions and recovered and non-recovered fractions $\left(S_{\mathrm{r}}\right.$ and $\left.S_{\mathrm{nr}}\right)$ for SE DP 360, SE DP 403 pulps and Avicel PH 101.

Table 4 Cellulose II content of original samples, insoluble fractions and recovered fractions $\left(\mathrm{S}_{\mathrm{r}}\right)$ for SE DP 360, SE DP 403 pulps and Avicel PH 101.

\section{Figure legends}

Fig. 1 Centrifugation protocol in three steps to fractionate the insoluble fractions $\left(\mathrm{I}_{1}, \mathrm{I}_{2}, \mathrm{I}_{3}\right)$ and the clear solution fraction (S) in SE DP 360 and SE DP $403 / \mathrm{NaOH}$ $8 \%$ - water solutions.

Fig. 2 Optical microscopy images of the fraction $\mathrm{I}_{1}$ from the SE DP 360 and SE DP 403 / NaOH 8\% - water solutions. Ballooned fibres, highly swollen fibres, highly swollen sections and flat rings are observed.

Fig. 3 Optical microscopy images of the fraction $I_{2}$ from the SE DP 360 and SE DP 403 / $\mathrm{NaOH} \mathrm{8 \%} \mathrm{-} \mathrm{water} \mathrm{solutions.} \mathrm{Flat} \mathrm{rings} \mathrm{and} \mathrm{fragments} \mathrm{of} \mathrm{rings} \mathrm{are}$ observed.

Fig. 4 Optical microscopy images of the fraction $I_{3}$ from the SE DP 360 and SE DP 403 / NaOH 8\% - water solutions. Only small fragments 10-50 $\mu \mathrm{m}$ are observed. 
Fig. 5 TEM image of the clear solution fraction (S) from a SE DP 360 / $\mathrm{NaOH} \mathrm{8 \%}$ - water solution. Despite the optical microscopy observations do not show insoluble parts due to their small size and lack of optical contrast because of their very high swelling, a few small insoluble particles can be seen by TEM.

Fig. 6 Optical microscopy images of highly swollen fibres, sections and flat rings observed in a non-agitated SE DP 403 / NaOH 8\% - water solution. (a) direct light transmission; (b) between cross-polarizer.

Fig. 7 Optical microscopy images of highly swollen fibres, sections and flat rings. (a) bleached sulphite pulp / $\mathrm{NaOH} 8 \%$ - water solution; (b) PH Kraft pulp / $\mathrm{NaOH}$ $8 \%$ - water solution.

Fig. 8 Optical microscopy images of fraction I from a Avicel PH 101 / $\mathrm{NaOH} \mathrm{8 \%}$ - water solution, (a) in transmission light and (b) between cross-polarizer. Insoluble parts of about 1-10 $\mu \mathrm{m}$ are observed.

Fig. 9 Amounts of insoluble material in $\mathrm{NaOH} 8 \%$ - water solutions of $\mathrm{PH}$ Kraft pulp, bleached sulphite pulp, SE DP 403, non-agitated SE DP 403 and Avicel PH 101.

Fig. 10 Molecular weight distributions of original samples, insoluble fractions $\left(I_{1}\right.$, $\mathrm{I}_{2}, \mathrm{I}_{3}$ and I) and recovered fractions $\left(\mathrm{S}_{\mathrm{r}}\right)$ for SE DP 360, SE DP 403 wood pulps and Avicel PH 101.

Fig. 11 FTIR-ATR spectra of different fractions as compared to the most equivalent cellulose I / cellulose II composition reference spectrum.

Fig. 12 Schematic representation of the dissolution steps of wood pulp fibres in $\mathrm{NaOH} 8 \%$ - water. 
Tables

\begin{tabular}{|l|l|l|c|c|}
\hline Samples & Origin & Pre-treatment & DP & Fibre / particle diameter \\
\hline SE DP 403 & Spruce (Borregaard) & Sulphite + steam explosion (SE) 15 bars, 100 s & 403 & $15-30 \mu \mathrm{m}$ \\
\hline SE DP 360 & Spruce (Borregaard) & Sulphite + steam explosion (SE) 15 bars, 100 s & 360 & $15-30 \mu \mathrm{m}$ \\
\hline Avicel PH 101 & Wood origin unknown & Hydrolysis (MCC) & 170 & $5-100 \mu \mathrm{m}$ \\
\hline Bleached sulphite pulp & Spruce (Borregaard) & Sulphite & 400 & $15-30 \mu \mathrm{m}$ \\
\hline PH Kraft pulp & Mixed hardwood & Pre-hydrolysed (PH) kraft & 2000 & $15-30 \mu \mathrm{m}$ \\
\hline
\end{tabular}

Table 1 


\begin{tabular}{|l|l|c|}
\hline Samples & Fractions & Average amount \% \\
\hline \multirow{4}{*}{ SE DP 360 } & 3,5 \\
\cline { 2 - 3 } & $\mathrm{l}_{1}$, large insoluble parts & 6,4 \\
\cline { 2 - 3 } & $\mathrm{I}_{2}$, Medium insoluble parts & 3,8 \\
\cline { 2 - 3 } & $\mathrm{l}_{3}$, small insoluble parts & 58,2 \\
\cline { 2 - 3 } & $\mathrm{S}_{\mathrm{r}}$, recovered material from S & 28,1 \\
\cline { 2 - 3 } & $\mathrm{S}_{\mathrm{n}}$, non-recovered material from S & 4,7 \\
\hline \multirow{4}{*}{ SE DP 403 } & $\mathrm{I}_{1}$, large insoluble parts & 5,0 \\
\cline { 2 - 3 } & $\mathrm{I}_{1}$, Medium insoluble parts & 2,3 \\
\cline { 2 - 3 } & $\mathrm{I}_{3}$, small insoluble parts & 58,5 \\
\cline { 2 - 3 } & $\mathrm{S}_{\mathrm{r}}$, recovered material from S & 29,6 \\
\cline { 2 - 3 } & $\mathrm{S}_{\mathrm{n}}$, non-recovered material from S & 2,6 \\
\hline \multirow{3}{*}{ Avicel PH 101 } & $\mathrm{I}$, small insoluble parts & 67,3 \\
\cline { 2 - 3 } & $\mathrm{S}_{\mathrm{r}}$, recovered material from S & 30,1 \\
\cline { 2 - 3 } & $\mathrm{S}_{\mathrm{n}}$, non-recovered material from S & \\
\hline
\end{tabular}

Table 2 


\begin{tabular}{|c|c|c|c|c|c|c|c|}
\hline SE DP 360 & in $100 \mathrm{~g}$ & Glucan & Xylan & Mannan & Mannan / xylan & Glucan / mannan & Glucan / xylan \\
\hline Original wood pulp & 100,0 & 97,70 & 1,400 & 0,900 & 0,64 & 109 & 70 \\
\hline Insoluble fraction $I_{1}+I_{2}+I_{3}$ & 13,7 & 13,58 & 0,032 & 0,078 & 2,44 & 174 & 424 \\
\hline Recovered fraction $S_{r}$ & 58,2 & 57,90 & 0,116 & 0,175 & 1,50 & 332 & 497 \\
\hline Non-recovered fraction $S_{n r}$ & 28,1 & 26,22 & 1,252 & 0,647 & 0,52 & 41 & 21 \\
\hline SE DP 403 & in $100 \mathrm{~g}$ & Glucan & Xylan & Mannan & Mannan / xylan & Glucan / mannan & Glucan / xylan \\
\hline Original wood pulp & 100,0 & 97,80 & 1,400 & 0,900 & 0,64 & 109 & 70 \\
\hline Insoluble fraction $I_{1}+I_{2}+I_{3}$ & 12,0 & 11,90 & 0,020 & 0,072 & 3,60 & 165 & 595 \\
\hline Recovered fraction $S_{r}$ & 58,5 & 58,27 & 0,117 & 0,176 & 1,50 & 332 & 498 \\
\hline Non-recovered fraction $S_{n r}$ & 29,6 & 27,63 & 1,263 & 0,652 & 0,52 & 42 & 22 \\
\hline Avicel PH 101 & in $100 \mathrm{~g}$ & Glucan & Xylan & Mannan & Mannan / xylan & Glucan / mannan & Glucan / xylan \\
\hline Original sample & 100,0 & 97,10 & 1,600 & 1,200 & 0,75 & 81 & 61 \\
\hline Insoluble fraction I & 2,55 & 2,52 & 0,0051 & 0,020 & 3,92 & 126 & 494 \\
\hline Recovered fraction $S_{r}$ & 67,33 & 67,00 & 0,135 & 0,135 & 1,00 & 496 & 496 \\
\hline Non-recovered fraction $S_{n r}$ & 30,12 & 27,58 & 1,460 & 1,045 & 0,72 & 26 & 19 \\
\hline
\end{tabular}

Table 3 


\begin{tabular}{|l|c|}
\hline Samples & $\%$ cellulose II \\
\hline SE DP 360 wood pulp & 0 \\
\hline SE DP 360 Fraction $I_{1}$ & 92 \\
\hline SE DP 360 Fraction $I_{2}$ & 88 \\
\hline SE DP 360 Fraction $I_{3}$ & 92 \\
\hline SE DP 360 Fraction $S_{r}$ & 94 \\
\hline SE DP 403 wood pulp & 0 \\
\hline SE DP 403 Fraction $I_{1}$ & 98 \\
\hline SE DP 403 Fraction $I_{2}$ & 96 \\
\hline SE DP 403 Fraction $I_{3}$ & 94 \\
\hline SE DP 403 Fraction $S_{r}$ & 97 \\
\hline Avicel PH 101 & 0 \\
\hline Avicel PH 101 - Fraction I & 100 \\
\hline Avicel PH101 - Fraction $S_{r}$ & 98 \\
\hline
\end{tabular}

Table 4 
Figures

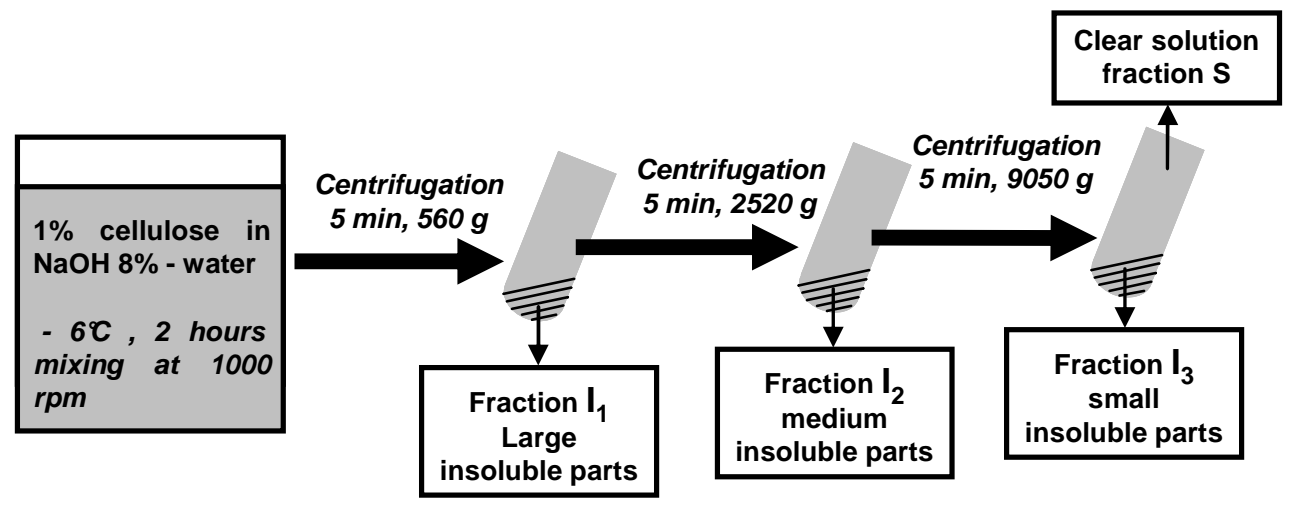

Fig. 1 


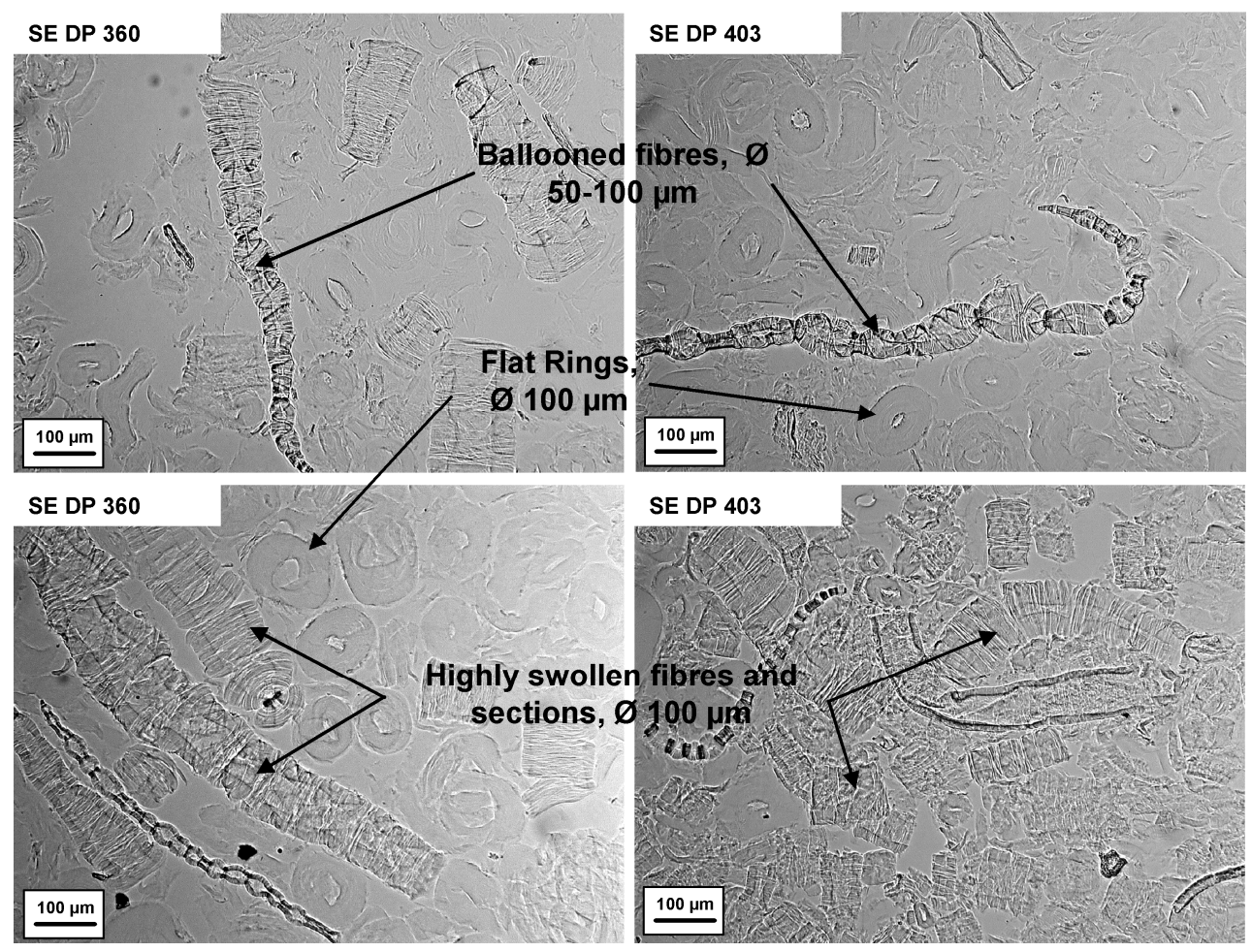

Fig. 2 


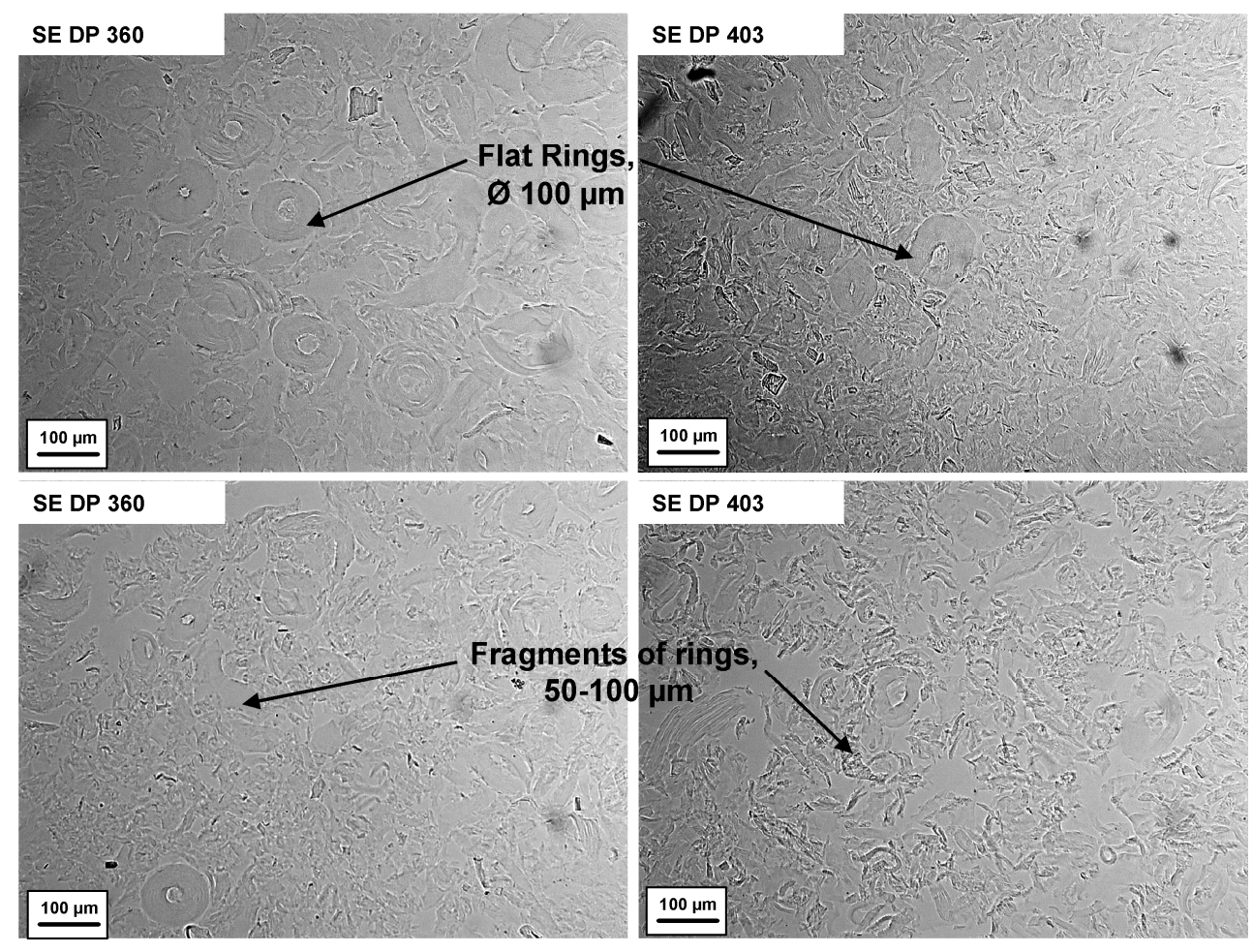

Fig. 3 


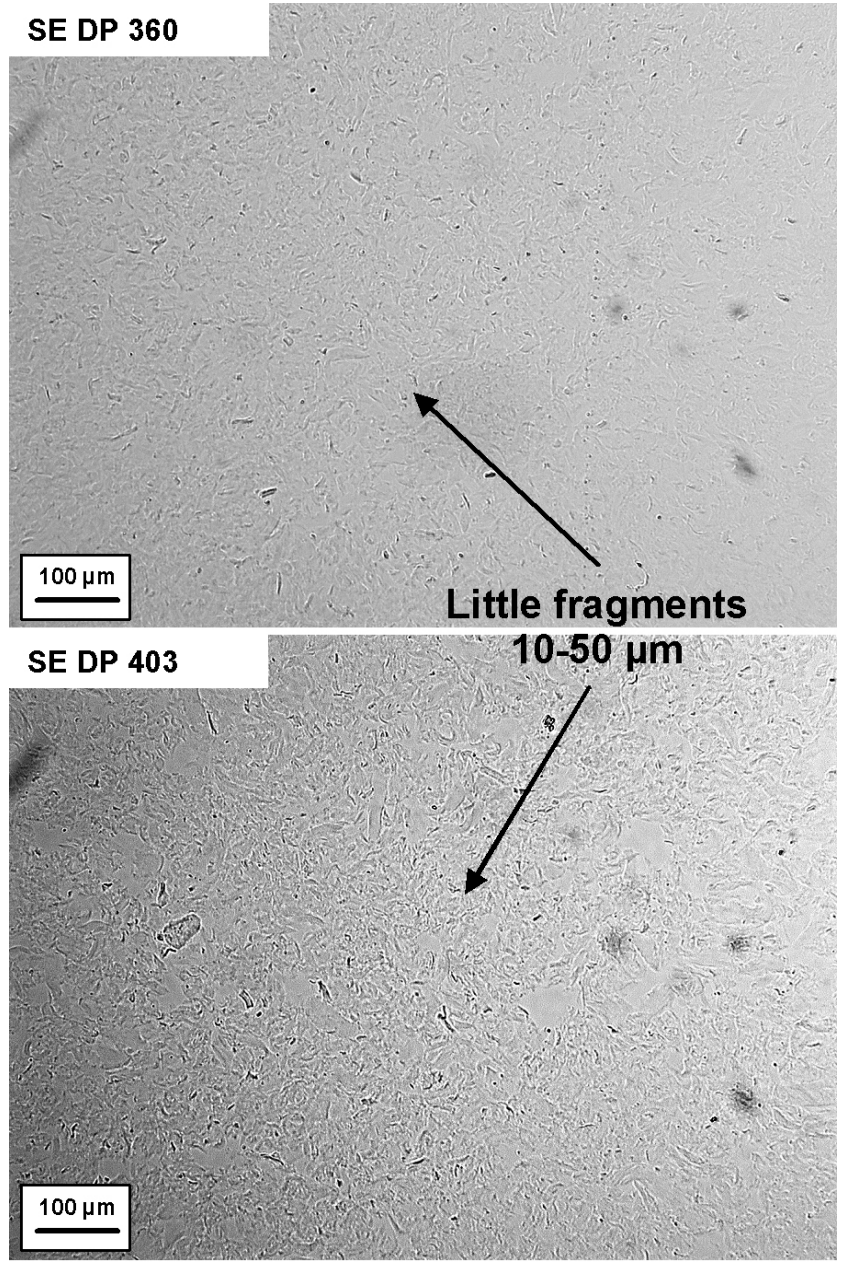

Fig. 4 


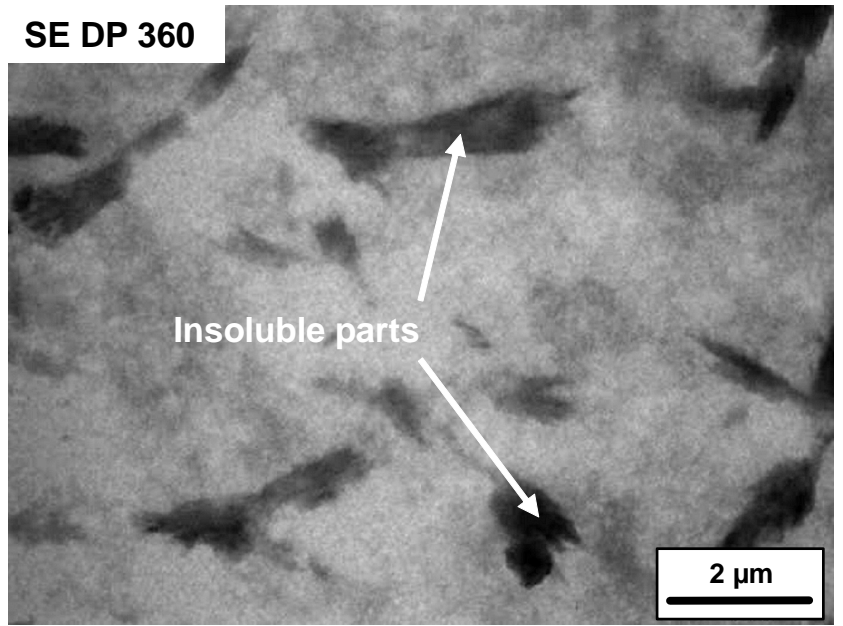

Fig. 5 


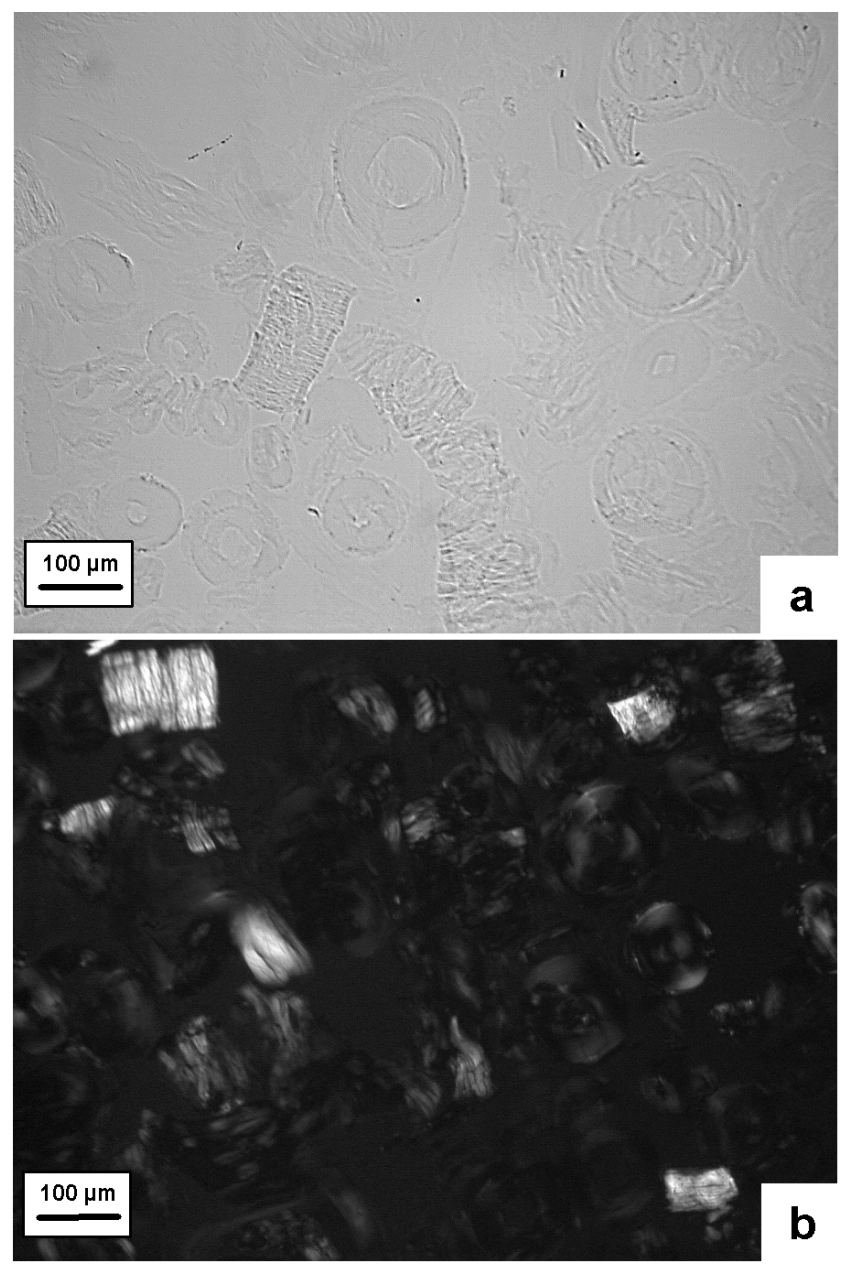

Fig. 6 

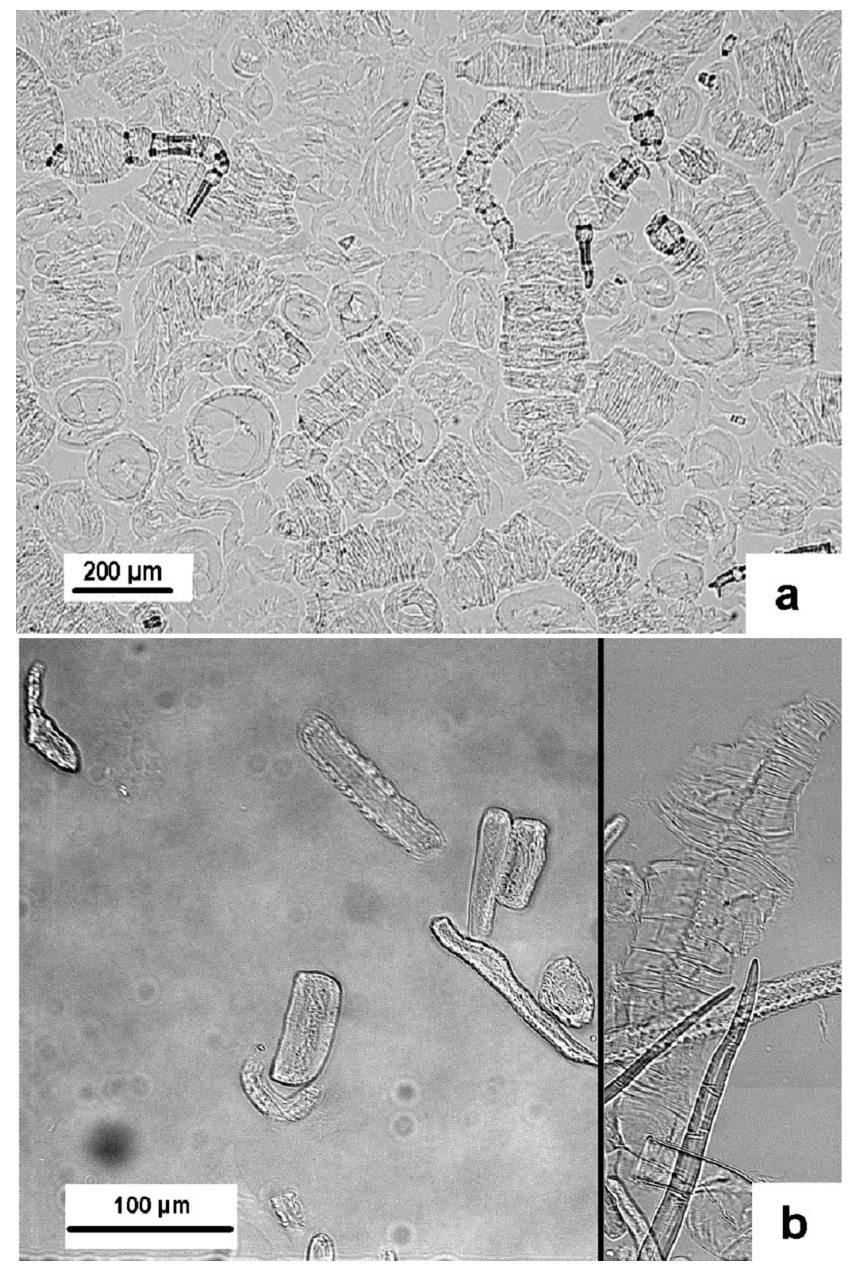

Fig. 7 


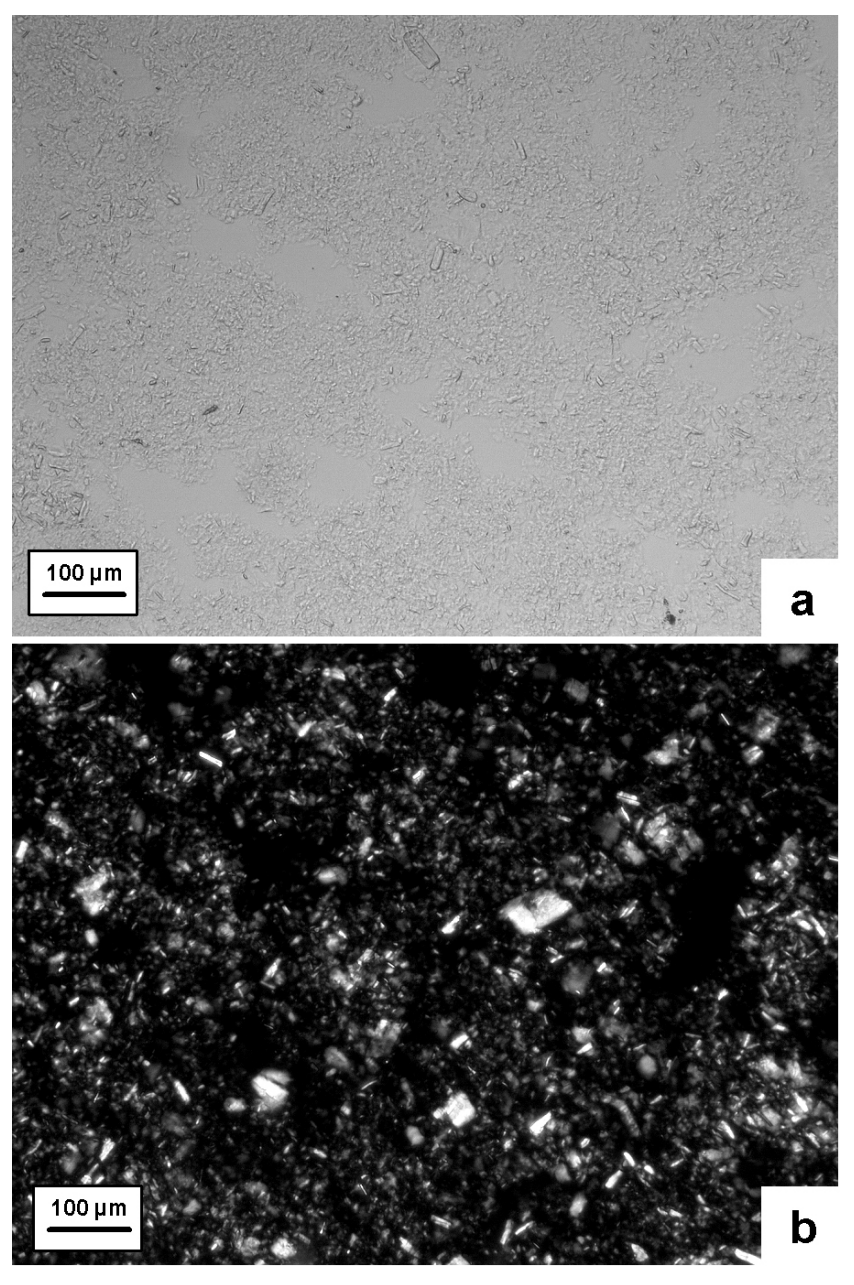

Fig. 8 


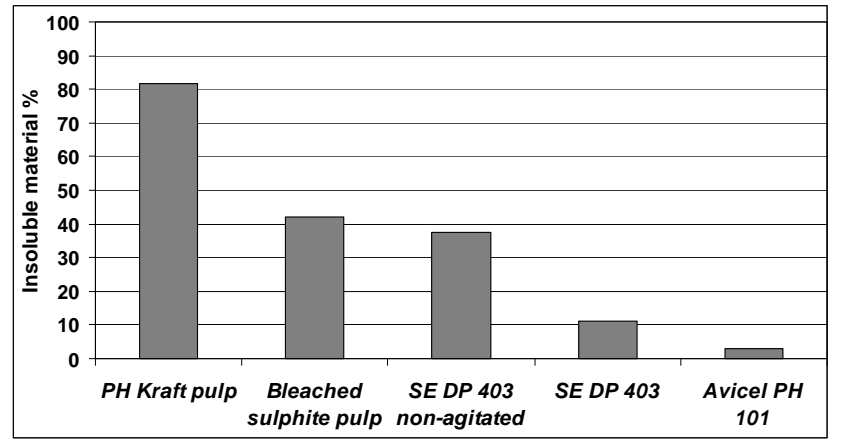

Fig. 9 

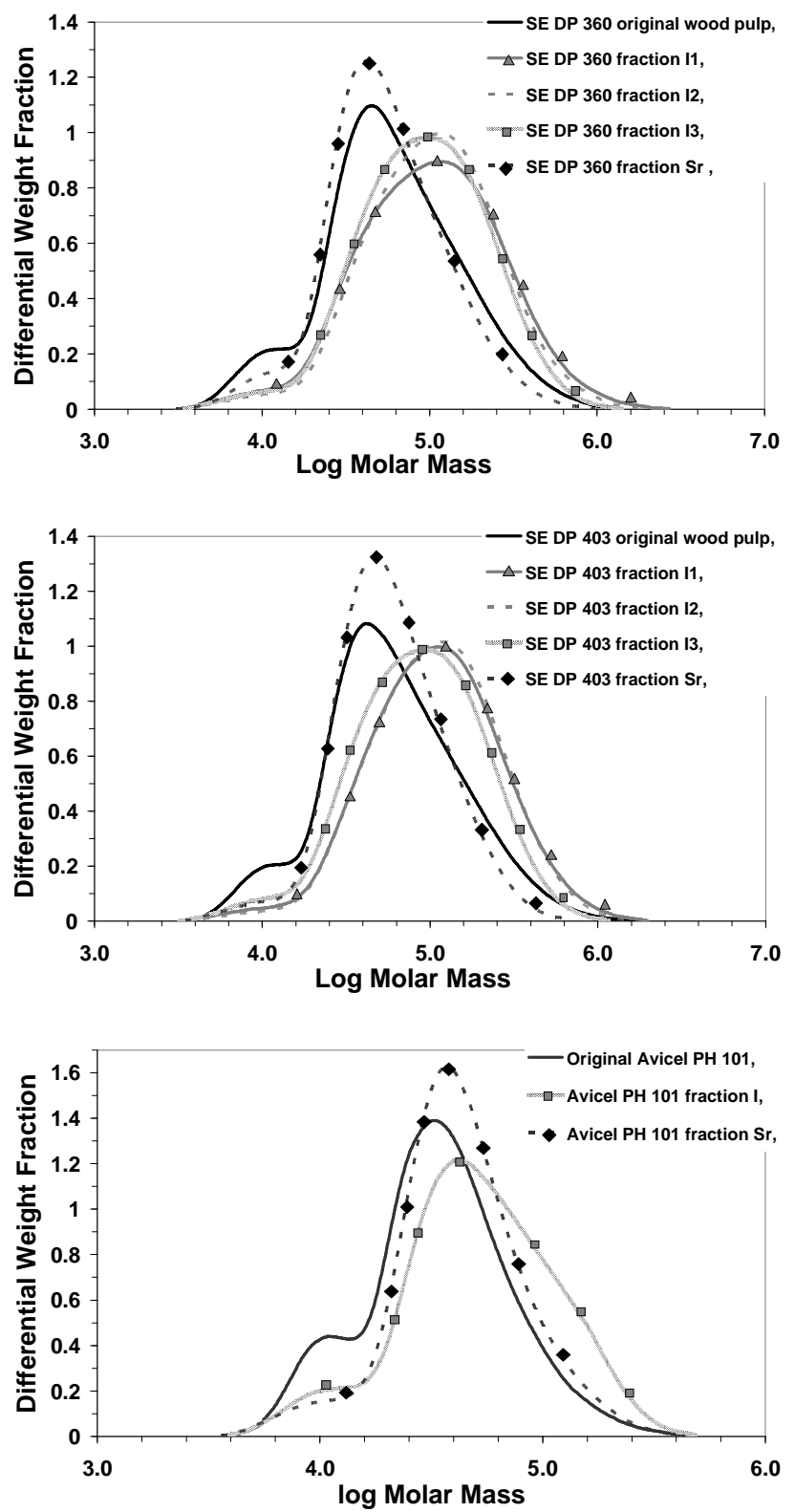

Fig. 10 


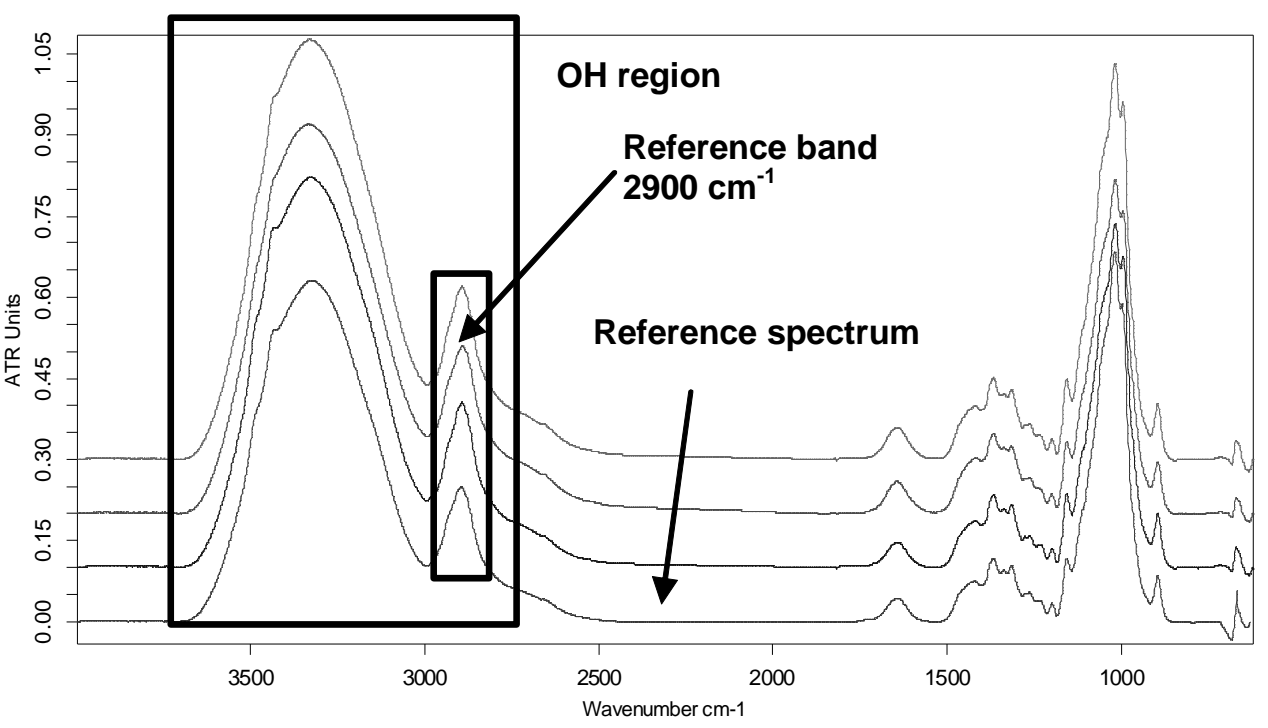

Fig. 11 


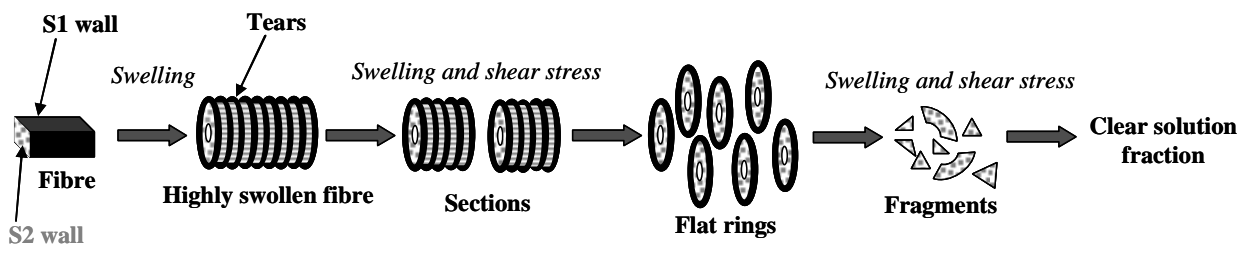

Fig. 12 